REPORT TITLE: Treatment of Produced Waters Using a Surfactant Modified Zeolite/Vapor Phase Bioreactor System

REPORT TYPE: Semi-Annual Progress

REPORT PERIOD: 1 October 2002 - 31 March 2003

PRINCIPAL AUTHORS: Lynn E. Katz, Kerry A. Kinney, R.S. Bowman, E.J. Sullivan REPORT ISSUE DATE: April 2003

DOE AWARD NUMBER: DE-FC26-02NT15461 


\section{Treatment of Produced Waters Using a Surfactant Modified Zeolite/Vapor Phase Bioreactor System}

Semi-Annual Progress Report

1 October 2002 - 31 March 2003

DOE AWARD NUMBER: DE-FC26-02NT15461

DISCLAIMER: This report was prepared as an account of work sponsored by an agency of the United States Government. Neither the United States Government nor any agency thereof, nor any of their employees, makes any warranty, express or implied, or assumes any legal liability or responsibility for the accuracy, completeness, or usefulness of any information, apparatus, product, or process disclosed, or represents that its use would not infringe privately owned rights. Reference herein to any specific commercial product, process, or service by trade name, trademark, manufacturer, or otherwise does not necessarily constitute or imply its endorsement, recommendation, or favoring by the United States Government or any agency thereof. The views and opinions of authors expressed herein do not necessarily state or reflect those of the United States Government or any agency thereof.

Lynn E. Katz

Project Manager 


\begin{abstract}
$\underline{\text { Abstract }}$
Co-produced water from the oil and gas industry accounts for a significant waste stream in the United States. It is by some estimates the largest single waste stream in the country, aside from nonhazardous industrial wastes. Characteristics of produced water include high total dissolved solids content, dissolved organic constituents such as benzene and toluene, an oil and grease component, and chemicals added during the oilproduction process. While most of the produced water is disposed via reinjection, some of them must be treated to remove organic constituents before the water is discharged. Current treatment options are successful in reducing the organic content; however, they cannot always meet the levels of current or proposed regulations for discharged water. Therefore, an efficient, cost-effective treatment technology is needed. Surfactantmodified zeolite (SMZ) has been used successfully to treat contaminated ground water for organic and inorganic constituents. In addition, the low cost of natural zeolites makes their use attractive in water-treatment applications. Our previous DOE research work (DE-AC26-99BC15221) demonstrated that SMZ could successfully remove BTEX compounds from the produced water. In addition, SMZ could be regenerated through a simple air sparging process. The primary goal of this project is to develop a robust SMZ/VPB treatment system to efficiently remove the organic constituents from produced water in a cost-effective manner.
\end{abstract}

This report summarizes work of this project from October 2002 to March 2003. In this starting stage of this study, we have continued our investigation of SMZ regeneration from our previous DOE project. Two saturation/stripping cycles have been completed for SMZ columns saturated with BTEX compounds. Preliminary results suggest that BTEX sorption actually increases with the number of saturation/regeneration cycles. Furthermore, the experimental vapor phase bioreactors for this project have been designed and are currently being assembled to treat the off-gas from the SMZ regeneration process. 


\section{Table of Contents}

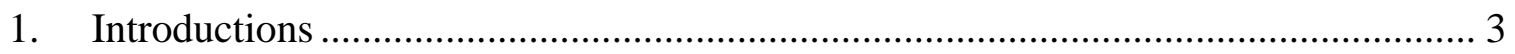

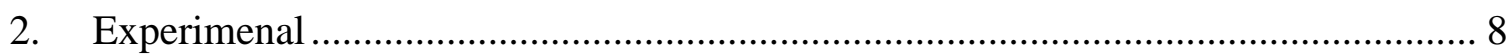

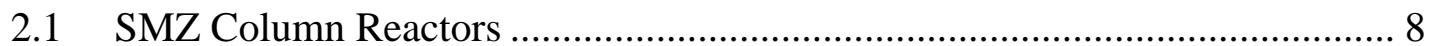

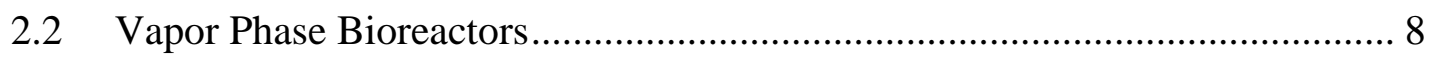

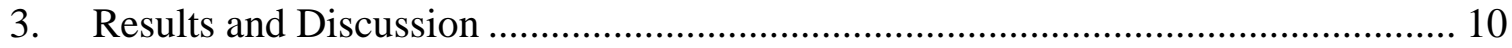

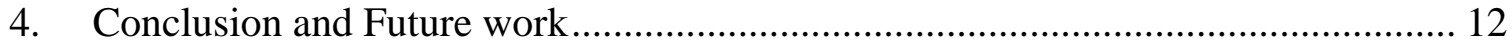

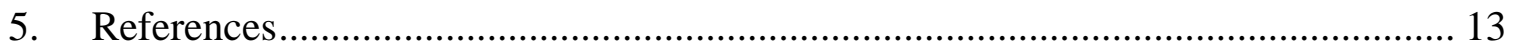




\section{Introduction}

Co-produced water from the oil and gas industry accounts for a significant waste stream in the United States. For each barrel (bbl) of oil produced, an average of $10 \mathrm{bbl}$ of water is produced for an annual total of about 3 billion tons (API, 1987). This is by some estimates the largest single waste stream in the country, aside from nonhazardous industrial wastes (Allen and Rosselot, 1994). Although as much as 95\% of this produced water is disposed via reinjection, the remaining amounts that are discharged on the surface are significant. Environmental and cost considerations make surface discharge of this water a more practical means of disposal in many instances. In addition, reinjection is not always a feasible option because of geographic, economic, or regulatory considerations. In these situations, it may be desirable, and often necessary from a regulatory viewpoint, to treat produced water before discharge. It may also be feasible to treat waters that slightly exceed regulatory limits for re-use in arid or drought-prone areas, rather than losing them to re-injection.

Characteristics of produced water include high total dissolved solids content (TDS), dissolved organic constituents such as benzene and toluene, an oil and grease component (which may be a dissolved product, an emulsion, or a separate phase) and chemicals added during the oil-production process. Current solutions to oil and grease restrictions and dissolved toxics (mostly from dissolved organic constituents) include ultrafiltration, advanced oil-water separation, hydrocyclones, chemical clarification, and gas flotation. Although many of these treatment options are successful at reducing the organic content of produced waters, they cannot always meet the levels of the current or proposed regulations (EPA NPDES, Federal Register V. 66, No. 14, pp.6607-6610). A need therefore exists to develop a cost-efficient treatment technology for the organic content in produced water. Such a technology is estimated to potentially maintain several thousand bbl of domestic production in a single impacted county. This can be multiplied by a factor of 10 to 20 for other counties that may be potentially impacted within the state of Texas alone. Therefore, the impact of regulation and the need for inexpensive treatment processes is considerable. 
Surfactant-modified zeolite (SMZ) is an innovative filtration/sorption medium that can meet these needs. This adsorbent can be cost-effectively produced using naturally occurring zeolites and commercially available surfactant. Zeolites are hydrated aluminum tectosilicates characterized by cage like structures having high internal and external surface areas, and high-cation exchange capacities. The low cost of natural zeolites ( $\$ 45$ to $\$ 60 /$ ton) makes their use attractive in water-treatment applications. In contrast to clays, however, natural zeolites can occur as millimeter- or-greater-sized particles and are free of shrink-swell behavior. As a result, zeolites exhibit superior hydraulic characteristics and are suitable for use in filtration systems (Breck, 1974).

Treatment of natural zeolites using cationic surfactants dramatically alters their surface chemistry. The large organic cations exchange readily with the native counterions on the external surface of the zeolite. This exchange produces a high-organic-carboncontent external surface while preserving a significant portion of the internal-surface cation-exchange capacity. The surfactant used to coat the external surface of zeolites in our previous work was hexadecyltrimethylammonium bromide (HDTMA). In addition to increasing the organic content of the sorbent, the HDTMA cation also produces the unique property of oxyanion sorption under high loading levels. Thus, the adsorbent produced can remove cationic, anionic and organic constituents of produced water.

A key feature required for successful application of SMZ to the treatment of produced water is regeneration of the SMZ. Although SMZ is not as efficient as activated carbon in removing highly soluble organics such as benzene, studies completed by the PIs indicate that SMZ can be readily regenerated for BTEX compounds simply by sparging ambient air through the SMZ column. This process generates a moist air stream contaminated with relatively low concentrations of volatile organic compounds (VOCs) including BTEX. Because these VOCs are biodegradable and present in dilute concentrations, a vapor phase bioreactor can be used to destroy the pollutants generated in the SMZ regeneration step. In vapor phase bioreactors (VPBs), microorganisms growing on a fixed packing media are used to biodegrade organic pollutants found in the waste gas stream being treated. Products of the biodegradation include carbon dioxide, water and new biomass. This technology has several advantages for this application including high destruction efficiencies for many VOCs including BTEX compounds, low 
operating costs and minimal generation of undesirable byproducts (van Groenestijn and Hesselink, 1993; Deshusses and Webster, 2000). For instance, removal efficiencies of greater than $90 \%$ can be achieved at BTEX loads below $96 \mathrm{~g} / \mathrm{m}^{3}$ packinghr, (Lu, et al., 2000). The hydrocarbon load produced from the SMZ regeneration step is far below this value and thus high removal efficiencies are expected. In fact, VPBs were originally developed to treat waste gas streams containing low concentrations of VOCs and this is considered the ideal application for the VPB technology (Leson and Winer, 1991; Leson and Smith, 1997; van Groenestijn and Hesselink, 1993.).

Several VPB configurations are possible for the SMZ application including a biofilter configuration with a stationary liquid film containing nutrients and biomass. Biofilters are often packed with a natural media such as peat or compost which is biologically active and can provide nutrients to the microorganisms growing on the packing material. Synthetic packing materials such as silicon oxide pellets have also been used successfully in biofilters (Song and Kinney, 2000; Woertz, et. al, 2001) and allow greater control over key operating parameters such as nutrient supply, biomass distribution and microbial composition of the biofilm. Biotrickling filters are an alternative VPB configuration in which a liquid nutrient phase is continuously recirculated throughout a synthetic packing material. Microorganisms living in the biofilm attached to the packing material as well as suspended in the recirculating liquid phase are responsible for the degradation of the VOC pollutants. Both VPB configurations have been used successfully to treat waste gas streams containing BTEX and other volatile hydrocarbons and hybrid systems which contain both biofilter and biotrickling filter design elements are also currently under development for treating complex VOC mixtures (Song, et. al, 2002).

Although VPBs are an attractive treatment for the SMZ regeneration step, several issues will need to be investigated to ascertain the effectiveness of the SMZ/VPB combination. For instance, the presence of multiple VOCs in the inlet gas stream can lead to competitive inhibition between the VOC substrates (Deeb and Alvarez-Cohen, 1999; Deshusses et al., 1995). In addition to BTEX compounds, the air stream generated from the SMZ regeneration step will include other volatile hydrocarbons. Vapor phase bioreactors have the ability to treat complex hydrocarbon streams such as those generated 
from the remediation of gasoline-impacted sites (Jutras, et al., 1997) but interactions between the VOC components of the waste gas stream must be determined for a particular application. For instance, the PIs have observed in a biofilter packed with synthetic media that the presence of toluene can inhibit the degradation of xylene; however, once the toluene has been degraded in the front end of the bioreactor, the remaining aromatic constituents can be successfully degraded. The PI's research team has also recently observed that such VOC degradation interactions may be mediated by the nitrogen availability in the biofilm. In the presence of excess nitrogen, for instance, toluene, xylene and ethylbenzene can be degraded simultaneously but under nitrogen limitation, xylene degradation is reduced (Song et al., 2002). The effect of nitrogen on VPB performance has been studied by the PI and others and is an important operating parameter for the system (Kinney et al., 1999; Moe and Irvine, 2001).

Another issue to consider for the SMZ/VPB combined treatment system is that VPBs prefer a continuous feed stream and thus the operation of the SMZ/VPB system will need to be optimized to match SMZ regeneration and VPB feed cycles. For instance, in a recent study completed by the PIs, it was determined that a VPB supplied with a very small but continuous VOC feed achieved much higher removal efficiencies following restart of the bioreactor than did a system that was simply shut off and not provided any VOCs during the shutdown period (Park and Kinney, 2001). Finally, it is important to note that although many of the organic constituents found in produced water are biodegradable, the high salinity of produced water prevents direct biological treatment of the produced water. Furthermore, direct air stripping of the produced water phase will not remove the less volatile organic contaminants. Thus, treatment of the water via SMZ followed by treatment of the regeneration gas via a VPB allows the removal of organic and inorganic constituents and ultimate destruction of the VOCs without the problems associated with high salinity and the presence of heavier organic species.

The primary goal of this project is to develop a robust SMZ/VPB treatment system to efficiently remove the organic constituents from produced water in a costeffective manner. Although both SMZ and VPB technologies have been developed separately by the PIs, a series of laboratory and field scale experiments are required to optimize the SMZ/VPB combination specifically for produced water. Once the air 
sparging/VBP system has been developed for the primary regeneration of the volatile constituents, a secondary regeneration step will be required on an infrequent basis for the non-volatile hydrophobic constituents. Evaluation, selection and design of the secondary regeneration method for the non-volatile organic constituents will be a key component to the success of the project. 


\section{Experimental}

\subsection{SMZ Column Reactors}

The zeolite used in this study is a natural clinoptilolite-rich zeolite from the St. Cloud mine near Winston, NM with an internal cation exchange capacity (CEC) of 800 $\mathrm{meq} / \mathrm{kg}$, and an external cation exchange capacity (ECEC) of 90-110 meq/kg. The zeolite was ground and screened to grain size of $80 \times 100$ mesh $(0.18$ to $0.15 \mathrm{~mm})$ for the laboratory columns. The SMZ was prepared by treating zeolite with HDTMA-Cl in the proportions of $60 \mathrm{~g}$ of zeolite with $180 \mathrm{~mL}$ of a $0.10 \mathrm{M}$ HDTMA-Cl solution. After shaking at $25^{\circ} \mathrm{C}$ for $24 \mathrm{hr}$, the HDTMA-zeolite was rinsed with two $180 \mathrm{~mL}$ aliquots of Millipore ${ }^{\circledR}$ water and air dried. The zeolite, however, was used without pretreatment prior to HDTMA sorption and the subsequent sorption experiments to more closely mimic expected field use.

Four laboratory columns were packed with 80x100 mesh SMZ for the regeneration study. After saturation with organic-free water having the same ionic composition as the produced water, a tritium breakthrough curve was generated to evaluate the hydraulic properties of the SMZ. Then the columns were fed with produced water until full breakthrough of benzene and toluene occurred. The columns were airstripped until benzene and toluene levels in the sparge gas dropped to zero. The columns were resaturated with produced water and stripped again.

\subsection{Vapor Phase Bioreactors}

The experimental vapor phase bioreactors for this project have been designed and are currently being assembled to treat the off-gas from the SMZ regeneration process. The bioreactor design selected for this project is a hybrid system that combines features of both biotrickling filters and biofilters. Similar to a biotrickling filter, the experimental bioreactor will be packed with a synthetic media and nutrients such as nitrogen will be supplied externally via a liquid spray system. However, the nutrients will be sprayed over the bed for only 30 minutes per day instead of continuously as occurs in biotrickling filters. This periodic nutrient supply will minimize the liquid film on the packing material 
since excess water can hinder the mass transfer and ultimately the removal rate of relatively hydrophobic hydrocarbon pollutants. This design also allows easier control over bioreactor conditions and enables adjustments such as increased nitrogen supply or $\mathrm{pH}$ control to be accomplished easily. The experimental bioreactor is also similar to classical biofilters since the liquid phase will be essentially immobile throughout most of the bioreactor operation.

The experimental bioreactor column (I.D. $16 \mathrm{~cm}$ ) consists of three individual sections bolted together (Figure 1). Each section will be packed with polyurethane foam cubes $(1.5 \mathrm{~cm})$ to a height of approximately $19 \mathrm{~cm}$. Polyurethane foam is attractive for this application since it has a relatively high surface area, is light weight and can be compressed to remove excess biomass. A $2 \mathrm{~cm}$ plenum located between each packed section will allow for gas sampling and redistribution of the contaminant stream between sections. This design has been used successfully in previous experiments to treat VOC mixtures containing both hydrophobic and hydrophilic constituents.

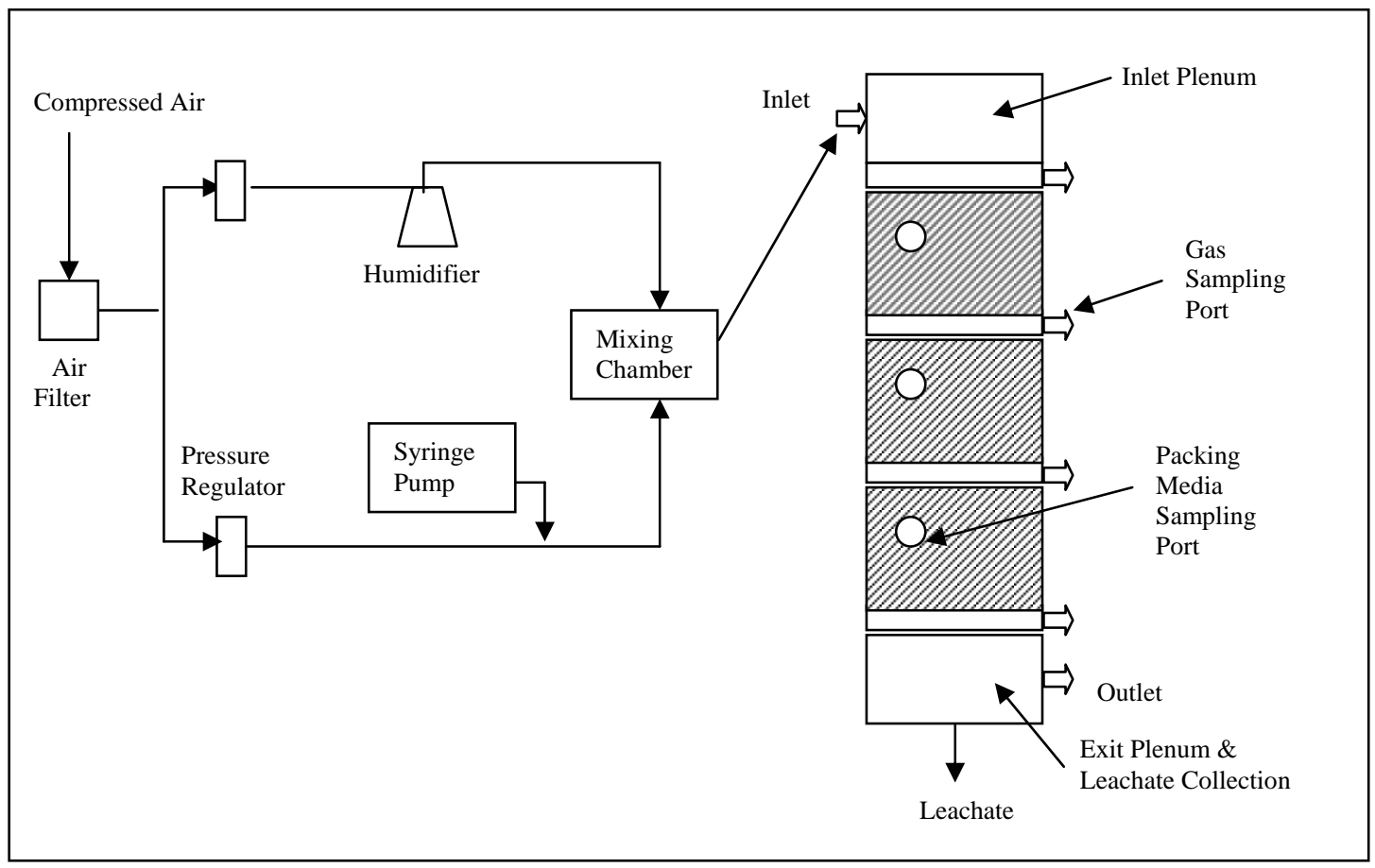

Figure 1. schematic diagram of experimental vapor phase biofilter design 


\section{Results and Discussion}

During the first six months of this project, we focused on work in Phases 1 and 2 of this project as stated in our project proposal. A major portion of the Phase I work is being conducted at the New Mexico Institute of Mining and Technology (NMT). The work is currently focusing on designing the SMZ column and regeneration process. The NMT Mexico group has completed several SMZ saturation/regeneration cycles to characterize BTEX retention capacity in the column reactors. Earlier experiments were performed using BTEX dissolved in $0.005 \mathrm{M} \mathrm{CaCl}_{2}$ solution. Currently, column sorption/regeneration experiments using produced water from the Crystal Solutions facility in Wyoming are being investigated. This is the same facility where a SMZ/produced water pilot test was performed in 2002. To date two saturation/stripping cycles have been completed. We plan a total of approximately ten cycles. Prior to the sixth cycle, new tritium breakthrough curves will be prepared to characterize hydraulic changes in the SMZ over time. Two of the four columns will then be sacrificed and the SMZ removed for BTEX batch sorption isotherm experiments. The BTEX isotherms for the regenerated SMZ will be compared to those for virgin SMZ. Preliminary results suggest that, due to sorption of high molecular weight components on the SMZ, BTEX sorption actually increases with the number of saturation/regeneration cycles.

Experimental studies for Phase 2 are underway at the University of Texas at Austin for designing vapor phase bioreactors that will treat the off-gas produced during regeneration of the SMZ column. We have designed and assembled a hybrid system that combines features of both biotrickling filters and biofilters and will test this system in conjunction with SMZ regenerating process.

We have also characterized the produced water composition (from the Wyoming test site) for organic compounds and their concentrations as part of the effort in Phase 1 (Table 1). The analytical results showed that in addition to the BTEX compounds, phenol and naphthalene compounds provided significant contributions to the organic content. These results will be employed to select the synthetic water for VPB and regeneration studies. 
Table 1. Produced Water Composition

\begin{tabular}{lc}
\hline Organic Compound & Concentration $(\mathrm{mg} / \mathrm{L})$ \\
\hline Benzene & 20,000 \\
Toluene & 35,000 \\
Ethylbenzene & 25,000 \\
o-Xylene & 5000 \\
m-Xylene & 5000 \\
p-Xylene & 5000 \\
phenol & 1000 \\
2-methylphenol & 1000 \\
4-methylphenol & 1000 \\
2,4-dimethylphenol & 500 \\
naphthalene & 250 \\
2-methylnaphthalene & 500
\end{tabular}




\section{Conclusions and Future work}

We found that in addition to BTEX, phenol and naphthalene compounds were also important organic pollutants in the produced water from the Wyoming test site. This information will be implemented to determine the composition of the influent stream for the vapor phase bioreactors that have been assembled in the mean time. For the SMZ regeneration phase, our preliminary results showed that BTEX retention in the SMZ column reactors increases with the number of regeneration cycles.

For the next six months of this project, we will focus on the following tasks to complete the proposed work in Phase 1 and Phase 2 of this project.

i) Synthetic waters with different organic constituents as well as produced water will be fed into the SMZ reactors. Organic contents of both influent and effluent will be analyzed to determine the retention capacity in SMZ column reactors. Based on the composition of organic compounds in the produced water, phenols and naphthalenes will be added into the synthetic waters in addition to the BTEX compounds.

ii) Regeneration will be conducted using high $\mathrm{pH}$ solutions for regeneration of nonvolatile components and an air-sparging process for volatile components. The optimal conditions for the regenerating process will be evaluated and the ultimate useful life of SMZ will be determined.

iii) Several hydrocarbon-degrading microbial cultures will be evaluated for the efficacy of degrading VOCs. Experiments will also be conducted to optimize a slip feed system to maintain VPB performance during periodic operation.

iv) To assess the performance of VPBs, the composition and relative concentrations of key pollutants will be systematically varied. Laboratory scale experiments of VPBs will be conducted first with a simple BTEX mixture, then with a more complex waste gas stream representing the VOCs expected during the SMZ regeneration step. 


\section{References}

API, 1987. Oil and gas industry exploration and production wastes.

Breck, D. W., 1974. Zeolite molecular sieves: structure, chemistry, and use. John Wiley and Sons, New York, 771 p.

Deeb, R. A. and Alvarez-Cohen, L (1999) "Temperature Effects and Substrate Interactions During the Aerobic Biotransformation of BTEX Mixtures by TolueneEnriched Consortia and Rhodococcus rhodochrous." Biotechnol. Bioeng., vol. 62, no. 5, 526-536.

Deshusses, M. A. and Webster, T. S. (2000) "Construction and Economics of a Pilot/FullScale Biological Trickling Filter Reactor for the Removal of Volatile Organic Compounds from Polluted Air." J Air Waste Manage. Assoc., vol. 50, no. 11, 1947-1956,

Deshusses, M. A., Hamer, G. and Dunn, I. J. (1995) "Behavior of Biofilters for Waste Air Biotreatment." Environ. Sci. Technol., vol. 29, 1048-1058.

Jutras, E. M., Smart, C. M., Rupert, R., Pepper, I. L. \& Miller, R. M. (1997) "Field-Scale Biofiltration of Gasoline Vapors Extracted from Beneath a Leaking Underground Storage Tank." Biodegradation, vol. 8, 31-42.

Kinney, K. A., Loehr, R. C. and Corsi, R. L. (1999) "Vapor-Phase Bioreactors: Avoiding Problems Through Better Design and Operation." Environ. Prog., vol. 18, no. 3, 222-230.

Leson, G. and Smith, B. J. (1997) "Petroleum Environmental Research Forum Field Study on Biofilters for Control of Volatile Hydrocarbons." J. Environ. Eng., vol. 123, no. 6, 556-562,

Leson, G. and Winer, A. M. (1991) "Biofiltration: An innovative Air Pollution Control Technology for VOC Emissions," J. Air Waste Manage.Assoc., vol. 41, no. 8, 1045-1054,

Lu, C., Chu, W. and Lin, M. 2000. "Removal of BTEX Vapor from Waste Gases by a Trickle Bed Biofilter,” J. Air \& Waste Manage. Assoc. 50: 411-417.

Moe, W. M. and Irvine, R. L (2001) "Effect of Nitrogen Limitation on Performance of Toluene Degrading Biofilters." Water Res., vol. 35, no. 6, 1407-1414

Park J, Kinney KA. (2001) "Evaluation of Slip Feed System for Vapor-Phase Bioreactors." J. Environ. Eng., vol. 127, no. 11, 979-985.

Song, J. and Kinney, K. A. (2000) "Effect of Vapor-Phase Bioreactor Operation on Biomass Accumulation, Distribution, and Activity." Biotechnol. Bioeng., vol. 68, no. 5, 508-516. 
Song, J. and Kinney, K. A., Boswell, J. T. and John, P. C. (2002) "Performance of a Compost-Based Hybrid Bioreactor for the Treatment of Paint Spray Booth Emissions." In Proceedings of the Air \& Waste Management Association's Annual Meeting \& Exhibition, 2002

van Groenestijn, J. W. \& Hesselink, P. G. M. (1993) "Biotechniques for Air Pollution Control," Biodegradation, vol. 4, 283-301.

Woertz, J. R., Kinney, K. A., McIntosh, N. D., Szaniszlo, P. J. (2001) "Removal of Toluene in a Vapor-Phase Bioreactor Containing a Strain of the Dimorphic Black Yeast Exophiala lecanii-corni." Biotechnol. Bioeng., vol. 75, no. 5, 550-558. 